\title{
Validation of the Sinhala translation of Edinburgh Postnatal Depression Scale
}

\author{
Dhammica Rowel' ${ }^{1}$ Pushpa Jayawardena ${ }^{1}$ and Neil Fernando ${ }^{2}$ \\ (Index words: Antenatal and postnatal depression, criterion validity, reliability)
}

\begin{abstract}
Introduction To determine the validity and reliability of the Sinhalese translation of the Edinburgh Postnatal Depression Scale (EPDS) as a screening instrument to detect antenatal and postnatal depression.

Method Two hundred and sixty five antenatal women and 204 postnatal women attending the field polyclinics of the Medical Officer of Health $(\mathrm{MOH})$ area Kolonnawa participated in the study. Psychiatric diagnosis based on ICD 10 Classification of Mental and Behavioural Disorders was the 'gold standard' and the receiver operating characteristic (ROC) analysis was used to evaluate the test performance of the translated EPDS for antenatal and postnatal depression separately. Reliability was tested using test-retest method and computing intra-class correlation coefficients.
\end{abstract}

Results A cut-off score of 9 was selected as the best to screen for depression, in antenatal (sensitivity $90.7 \%$ and specificity $86.8 \%$ ) and postnatal women (sensitivity $89.9 \%$ and specificity $78.9 \%$ ). The intra-class correlation coefficients of the instrument for antenatal and postnatal women were $0.95(95 \% \mathrm{Cl}=0.82-0.99)$ and $0.96(95 \%$ $\mathrm{Cl}=0.86-0.99)$.

Conclusion The Sinhalese translation of the EPDS is a valid and a reliable instrument to screen for depression in antenatal and postnatal women in Sri Lanka.

\section{Introduction}

In many South Asian countries, antenatal and postnatal depression are reaching epidemic proportions $[1,2]$ warranting community level public health action such as screening, early detection and treatment.

The EPDS was developed [3] to identify women experiencing postnatal depression. Since then the effectiveness of EPDS as an instrument to measure antenatal depression also has been confirmed [4]. The EPDS has been translated from the original English version [3] to south east Asian languages validated, and used successfully $[1,2]$. Such studies have shown variability in sensitivity, specificity and the optimal cut-off scores for prenatal and postnatal depression [1, 4]. Validation of the EPDS in a given population is essential before it can be used as a screening instrument.

\section{Method}

This study was done over a period of 4 months in 2003, in the field polyclinics of the $\mathrm{MOH}$ area Kolonnawa in Sri Lanka. Women who could read and understand Sinhalese were invited to participate when they attended the clinic for their regular antenatal appointments (at 34 weeks of period of amenorrhoea) or when the postpartum women (6 weeks after delivery) attended the family planning or child welfare clinics.

An expert panel reviewed the original English version of the EPDS for comprehensiveness and appropriateness of the questionnaire before translation. The questionnaire was translated using the 'translation back translation technique' [5]. The semantics of the words and the general structure of the final Sinhalese version of the instrument were in close agreement with those of the original English version. The alternate form reliability of the instrument was checked following the pre-test of the translated version.

The 'gold standard' for diagnosis of depression was the diagnosis made by the psychiatrist based on the clinical examination and ICD 10 Classification of Mental and Behavioural Disorders [6]. All participants completed the self-administered Sinhalese version of the EPDS first and were examined thereafter on the same clinic day by the psychiatrist who was blind to the EPDS score.

For the validation of EPDS for antenatal and postnatal women a sensitivity of $85 \%$ and a specificity of $80 \%$ were aimed at, which were the values targeted in most of the other studies on validation of this instrument [3, 7]. According to the sample size calculation based on the above sensitivity and specificity, 49 depressed women and 61 non-depressed women each in antenatal and postnatal groups were required in the sample. Two hundred and sixty five antenatal women and 204 postnatal women had to be examined to identify 49 depressed women in each category.

The best cut-off scores for screening antenatal and postnatal depression were determined using the receiver operation characteristics (ROC) curves and evaluating the sensitivity and specificity at those cut-off scores.

${ }^{1}$ Department of Community and Family Medicine, Faculty of Medicine, University of Kelaniya and ${ }^{2}$ Mental Hospital, Angoda, Sri Lanka.

Correspondence: DR, e-mail <edrowel@sltnet.lk> and <Dhammica.Rowel@nuigalway.ie>. Received 22 June, and revised version accepted 11 November 2007. Conflicts of interest: none declared. 


\section{Results}

\section{Criterion validity of EPDS for antenatal women}

The EPDS scores for antenatal women diagnosed as depressed by the Psychiatrist ranged from 5-23 (median = 13.0). Women diagnosed as having a normal mood had scores ranging from 0-17 (median $=5.0)$. The area under the ROC curve for antenatal women (figure 1) was 0.96 $(95 \% \mathrm{CI}=0.93-0.99 ; \mathrm{SE}=0.01)$. A score of ' 9 ' which gave a sensitivity of $90.7 \%(95 \% \mathrm{CI}=76.2 \%-96.4 \%)$ and a specificity of $86.8 \%(95 \% \mathrm{CI}=80.9 \%-90.9 \%)$ was selected as the best cut off to screen for depression in antenatal women. This gave a false positive rate of $12.2 \%(95 \% \mathrm{CI}=$ $8.3 \%-17.1 \%)$ and a false negative rate of $8.2 \%(95 \% \mathrm{CI}=$ $2.7 \%-18.5 \%)$. The Youden's index was 0.8 (95\% CI $=0.7$ $0.9)$. The likelihood ratios for positive and negative tests were $7.6(95 \% \mathrm{CI}=5.2-10.9)$ and $0.1(95 \% \mathrm{CI}=0.04-0.24)$.

\section{Criterion validity of EPDS for postnatal women}

The EPDS scores for the postnatal women who were diagnosed as depressed by the Psychiatrist ranged from $5-22($ median $=13)$. Women diagnosed as having a normal mood had scores ranging from $0-17($ median $=5)$. The area under the ROC curve for postnatal women (figure 2) was $0.94(95 \% \mathrm{CI}=0.91-0.98 ; \mathrm{SE}=0.02)$. A score of 9 which gave a sensitivity of $89.9 \%(95 \% \mathrm{CI}=74 \%-96.1 \%)$ and a specificity of $78.9 \%(95 \% \mathrm{CI}=70.6 \%-84.9 \%)$ was selected as the best cut-off to screen for depression in postnatal women. This gave a false positive rate of $19.4 \%(95 \% \mathrm{CI}=$ $13.7 \%-26.1 \%)$ and a false negative rate of $8.2 \%(95 \% \mathrm{CI}=$ $2.7 \%-18.5 \%)$. The Youden's index was $0.7(95 \% \mathrm{CI}=0.6$ 0.8 ). The likelihood ratios for positive and negative tests were $4.7(95 \% \mathrm{CI}=3.4-6.6)$ and $0.1(0.04-0.26)$ respectively.
In measuring the reliability of the EPDS, the intraclass correlation coefficient for antenatal and postnatal women were $0.95(95 \% \mathrm{CI}=0.82-0.99)$ and $0.96(95 \% \mathrm{CI}=$ 0.86-0.99) respectively, which demonstrated values closer to ' 1 '.

\section{Discussion}

Translation and validation of an instrument is an important procedure that appraises the reliability and validity of the instrument when a version other than the original is used.

Semantic equivalence has been particularly difficult to achieve in cross-cultural research. The essence of semantic equivalence is that the meaning of each item remains the same after translation into the language of each culture. The idioms used in a given culture, which may not impart the same meaning in another language, may seriously affect the respondent's view of the question posed and result in inaccurate responses [8]. This problem was detected and overcome by using suitable semantics.

Different indices of reliability may be used depending on the purpose of the measurement. For a predictive measure it is the intra-class correlation coefficient that is appropriate [9]. The intraclass correlation coefficient of 0.95 in antenatal women and 0.96 in postnatal women is considered highly reliable [10].

The best way of appraising validity is to find a criterion (a 'gold standard') that is known or believed to be close to the truth and to compare the results of the measure with this criterion. The validity of a screening test is measured by its ability to correctly categorise persons who have disease as test positive (sensitivity) and those

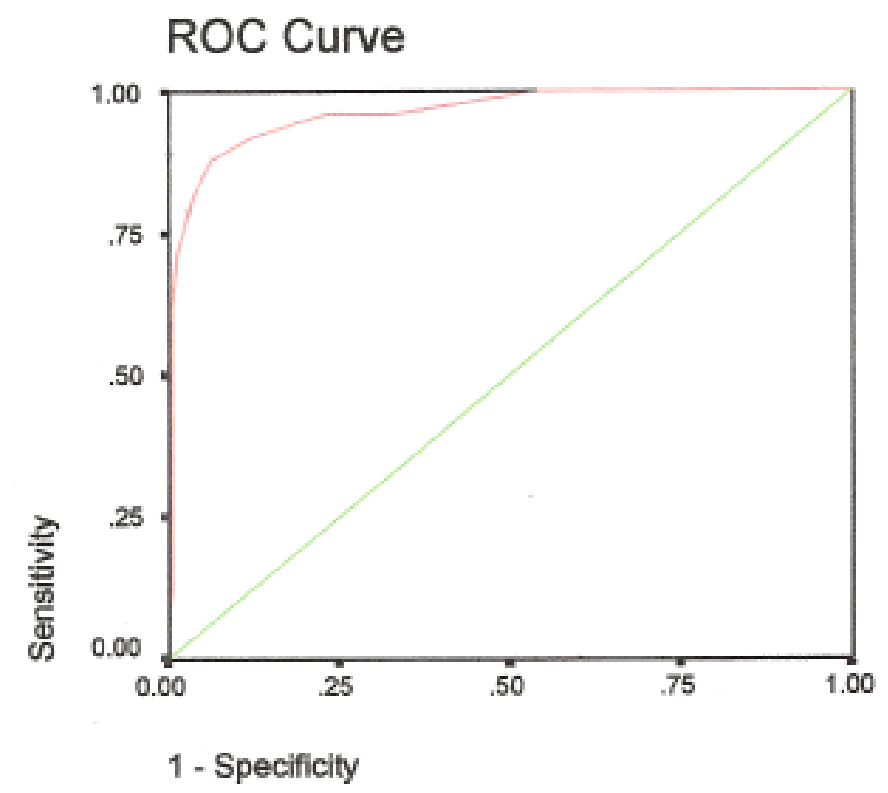

Figure 1. The receiver operation characteristic curve for antenatal women 


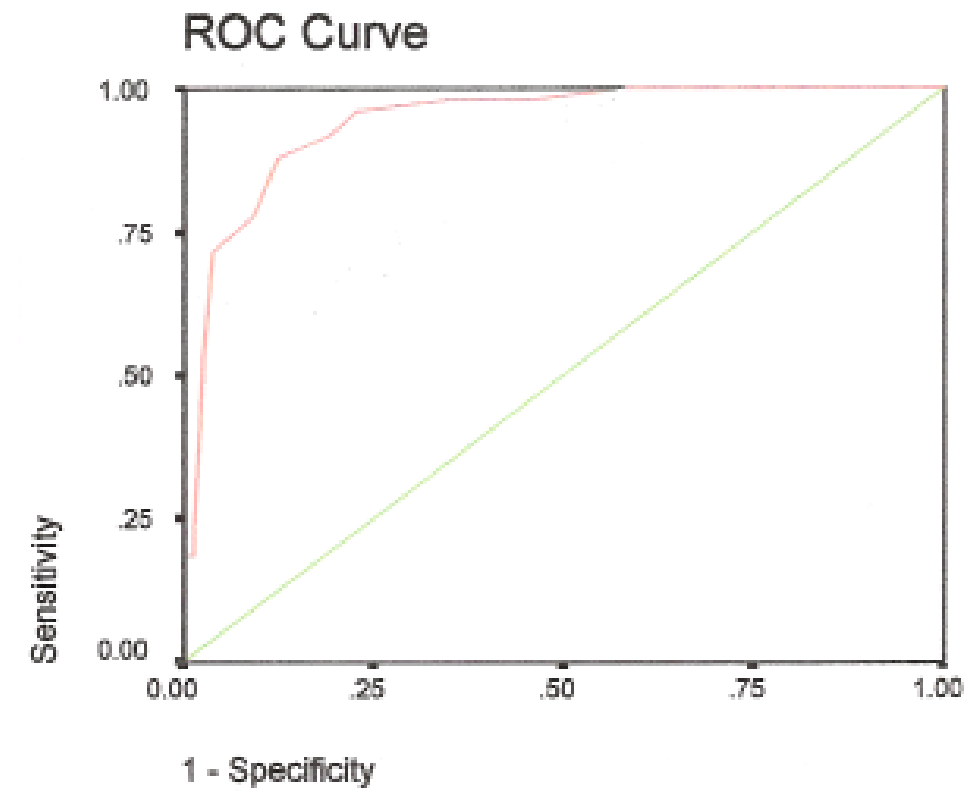

Figure 2. The receiver operation characteristic curve for postnatal women

without disease as test negative (specificity). The primary objective of a screening test is to detect disease early and reduce morbidity. In this specific instance of detecting depression, higher sensitivity as opposed to specificity was considered more appropriate to keep false negative rate at a minimal level.

A ROC plot is obtained by calculating the sensitivity and specificity of every observed data value and plotting sensitivity against 1 -specificity as in figures 1 and 2 . The accuracy of a test depends on how well the test separates the group being tested into those with and without the disease and is measured by the area under the ROC curve. ROC curves plotted for both antenatal and postnatal women in this instant had an area under the curve close to 1 indicating that the Sinhalese translation of the EPDS has a high discriminatory power [11].

In the original validation study of EPDS [3] for postnatal depression, with a threshold score of $12 / 13$, all women with major depression and two with probable major depression were correctly identified. That instrument had a sensitivity of $86 \%$ and specificity of $78 \%$. A similar cut-off point was found to detect major depression in Asian countries [1]. Cox and Holden [12] have recommended lowering the cut-off to $9 / 10$ in order to increase the detection rate if the EPDS is used in the first stage of screening in a community study. It was possible to achieve the same recommended cut-off score for the present study, which it is intended to be used as a community screening instrument.

\section{Conclusion}

The Sinhalese translation of the EPDS is a valid and a reliable instrument to screen for depression in antenatal and postnatal Sinhala women in Sri Lanka. The best cutoff score for the EPDS to screen for antenatal and postnatal depression is 9 .

\section{Acknowledgements}

The authors thank Dr Sumanatissa and Dr Sujeewana Amerasinghe, Registrars in Psychiatry, Mental Hospital, Angoda for assisting in the validation of the EPDS.

\section{References}

1. Patel V, Rodrigues M, DeSouza N. Gender, poverty and postnatal depression: a study of mothers in Goa, India. American Journal of Psychiatry 2002; 159: 43-7.

2. Rahman A, Iqbal Z, Harrington R. Life events, social support and depression in child birth: perspectives from a rural community in the developing world. Psychological Medicine 2003; 33: 1161-7.

3. Cox JL, Holden JM, Sagovsky R. Detection of postnatal depression: development of the 10-item Edinburgh Postnatal Depression Scale. The British Journal of Psychiatry 1987; 150: $782-6$.

4. Evans J, Heron J, Francomb H, Oke S, Golding J. Cohort study of depressed mood during pregnancy and after childbirth. British Medical Journal 2001; 323: 257-60.

5. Brislin RW. Back-translation for cross-cultural research. Journal of Cross-Cultural Psychology 1970; 1: 185-216.

6. The ICD-10 classification of mental and behavioural disorders. Clinical descriptions and diagnostic guidelines. Geneva: WHO; 1992: 119-23.

7. Murray L, Carothers AD. Validation of EPDS on a community sample. The British Journal of Psychiatry 1990; 157: 288-90. 
8. Flaherty JA, Gaviria FM, Pathak D, Mitchell T, Wintrob R, et al. Developing instruments for cross-cultural psychiatric research. The Journal of Nervous and Mental Disease 1988; 176: $257-63$.

9. Abramson JH, Abramson ZH. Survey Methods in Community Medicine, 5th ed. Edinburgh: Churchill Livingston; 1999.
10. Litwin MS. How to assess and interpret survey psychometrics. The Survey Kit 2. 2nd ed. London: Sage Publications, 2003.

11. Altman DG, Bland MJ. Diagnostic tests 3: receiver operating characteristic plots. British Medical Journal 1994; 309: 188.

12. Cox JL. Holden J. eds. Perinatal psychiatry: use and misuse of EPDS. London: Gaskell, 1994. 